\title{
Metales pesados asociados con las partículas atmosféricas y sedimentadas de superficies viales: Soacha (Colombia) ${ }^{1}$
}

Heavy metals associated with the atmospheric and sedimented particles from road surfaces: Soacha (Colombia)

\section{Partículas metais pesados associado atmosféricas e da su- perfície sedimentado road: Soacha (Colômbia)}

Recibido: octubre de 2012

Aprobado: diciembre de 2012
Carlos Alfonso Zafra Mejía ${ }^{2}$

Lina Gineth Rodríguez Chitiva ${ }^{3}$

Yulieth Andrea Torres Cabrera ${ }^{4}$

\section{Resumen}

La elevada densidad de tráfico e intensa actividad industrial en áreas urbanas genera impactos significativos en la salud pública. El objetivo de este artículo es estudiar la concentración de metales pesados asociados con el material atmosférico en suspensión (PM10 y PST) y el sedimento acumulado sobre superficies viales del municipio de Soacha (Colombia). Se determinó la concentración $(\mathrm{mg} / \mathrm{kg})$ de metales $(\mathrm{Pb}, \mathrm{Cd}$ y $\mathrm{Fe})$ por medio de espectrometría de absorción atómica de llama y de plasma, inductivamente acoplada con espectrometría de masas. Los resultados muestran una distribución similar de los elementos metálicos para las partículas en suspensión y sedimentadas de superficies viales. Las concentraciones de $\mathrm{Pb}$ y Cd asociadas con PM10 son en promedio 17,6 y 4,7 veces superiores a las detectadas en el sedimento vial, respectivamente. Las concentraciones de $\mathrm{Pb}$ superan los límites establecidos por inhalación, ingestión y contacto con el suelo de la legislación utilizada como referencia.

Palabras clave: metales pesados, PM10,salud pública, sedimento vial, Soacha.

\begin{abstract}
The high density of traffic and intense industrial activity in urban areas generates significant impacts on public health. The aim of this paper is to study the heavy metals concentration associated with the atmospheric material (PM10 and PST) and the sediment accumulated on road surfaces of Soacha (Colombia). It was determined the concentration (mg/ $\mathrm{kg}$ ) of metals $(\mathrm{Pb}, \mathrm{Cd}$ and $\mathrm{Fe}$ ) by means of atomic absorption flame and inductively coupled plasma mass spectrometry. The results show a similar distribution of the metallic elements for the suspended and deposited particles on road surfaces. The concentrations of $\mathrm{Pb}$ and $\mathrm{Cd}$ associated with PM10 are on average 17.6 and 4.7 times greater than those detected in the road sediment, respectively. The concentrations of $\mathrm{Pb}$ exceed the limits
\end{abstract}

\footnotetext{
Artículo de Investigación.

Facultad de Medio Ambiente, Universidad Distrital Francisco José de Caldas, Bogotá - Colombia. Contacto: czafra@udistrital.edu.co Facultad de Medio Ambiente, Universidad Distrital Francisco José de Caldas, Bogotá - Colombia. Contacto: linarodch@gmail.com Facultad de Medio Ambiente, Universidad Distrital Francisco José de Caldas, Bogotá - Colombia. Contacto: yatorresc@hotmail.com
} 
established by inhalation, ingestion and contact with the ground of the legislation used as reference.

Keywords: heavy metals, PM10, public health, road sediment, Soacha.

\section{Resumo}

A alta densidade de tráfego e atividade industrial intensa em áreas urbanas geram impactos significativos sobre a saúde pública. O objetivo deste trabalho é estudar a concentração de metais pesados associados com material atmosférica (PM10 e TSP) e sedimentos acumulados em estradas de Soacha (Colômbia). Determinar a concentração $(\mathrm{mg} / \mathrm{kg})$ de metais $(\mathrm{Pb}, \mathrm{Cd}$ e $\mathrm{Fe})$, por meio de absorção atómica de chama e indutivamente acoplado espectrometria de massa com plasma. Os resultados mostram uma distribuição semelhante de elementos metálicos para as partículas em suspensão e superfícies de estrada sedimentadas. As concentrações de $\mathrm{Pb}$ e Cd associados de PM10 são em média de 17,6 e 4,7 vezes maiores do que os encontrados em sedimentos do frasco, respectivamente. Concentrações de $\mathrm{Pb}$ exceder os limites fixados por inalação, ingestão e contato com o chão da legislação utilizada como referência.

Palavras-chave: metais pesados, PM10, saúde pública, sedimentos estrada, Soacha.

\section{Introducción}

A escala local se emiten a la atmósfera partículas y gases potencialmente nocivos que afectan la salud humana y el ambiente, y que en el largo plazo deterioran los recursos necesarios para el desarrollo ambiental de las áreas urbanas (OMS, 2004). En este sentido, se han reportado los metales pesados como parte de los contaminantes identificados en el aire y el suelo de las áreas urbanas (p.ej., Zafra et al., 2011; Pérez, 2005). Las emisiones de metales pesados a la atmósfera se asocian con fuentes naturales (p.ej., tormentas de arena e incendios forestales) y fuentes antropogénicas (i. e., fijas y móviles) (García, 2007).Estas últimas son las de mayor importancia en las áreas urbanas (Juda-Rezler et al., 2011).

Debido al carácter acumulativo y de permanencia de los metales pesados, estos se encuentran no solo en los diversos compartimentos ambientales (i. e., aire, agua, suelo, flora y fauna), sino que también se detectan en el organismo humano (Ferré-Huguet et al., 2007). Por lo tanto, los metales pesados pueden incorporarse al cuerpo humano a través del agua potable, ya sea por ingestión o por absorción dérmica durante una ducha o baño. Adicionalmente, pueden ser ingeridos, inhalados o absorbidos dérmicamente a partir de las partículas de polvo resuspendidas que provienen de los suelos de áreas contaminadas (p. ej., superficies viales); y pueden actuar como potentes tóxicos tanto para los seres humanos (cancerígenos) como para los ecosistemas, según cuáles sean sus vías y tiempo de exposición, la dosis absorbida y la naturaleza química del metal pesado (Ferré-Huguet et al., 2007). Desde el punto de vista de la salud pública, Amato et al. (2009) reportaron que la población urbana que vive o labora cerca de vías con una alta densidad de tráfico se encuentra en riesgo por la presencia de elementos metálicos.

Actualmente, la mayor parte del transporte de carga y de pasajeros entre Bogotá D.C. y el occidente y sur de Colombia transita por el municipio de Soacha y, específicamente, a través de su área urbana central, produciendo un impacto significativo en la calidad del aire y un deterioro del mobiliario urbano existente (AMS, 2009). Además, es uno de los municipios de Cundinamarca que por sus actividades industriales presentan un desmejoramiento de la calidad del ambiente y la salud pública (AMS, 2008). En este sentido, en la municipalidad se registran las mayores concentraciones 
de metales pesados en partículas respirables (Pachón et al., 2005). Es por esto que se hace necesario adelantar investigaciones para evaluar la presencia y estudiar el comportamiento de los metales pesados asociados con las partículas del material atmosférico en suspensión y el material acumulado sobre las superficies viales de la localidad. Lo anterior, con el objeto de que las instituciones públicas encargadas de la gestión de la contaminación ambiental en la municipalidad puedan diseñar, implementar y mejorar los sistemas de control de la contaminación por metales pesados.

La concentración de los contaminantes varía localmente por la convergencia o divergencia de los vientos, debido a que la masa es transportada (i. e., horizontal o verticalmente) hacia o desde un área determinada por la presencia de flujos turbulentos; así mismo, la concentración puede variar por fuentes de emisión de contaminantes y por sumideros locales (p.ej., transformaciones físicas y químicas y remoción por lluvia) (Bustos, 2004). Por lo tanto, la dispersión de los contaminantes a nivel local depende de parámetros meteorológicos como la velocidad y dirección del viento, la temperatura, el grado de estabilidad atmosférica y la altura de la capa de mezclado (Turtós et al., 2003).

Por otro lado, los mecanismos que transportan los contaminantes desde la atmósfera hacia la superficie subyacente se conocen como procesos de deposición; estos procesos constituyen el mayor sumidero de contaminantes atmosféricos (Olivares, 2001). Se pueden distinguir dos tipos: (i) la deposición húmeda se refiere a los procesos mediante los cuales los gases o partículas son removidos de la atmósfera por hidrometeoros ( $i$. e., gotas de nubes, neblina, lluvia y nieve) y, posteriormente, son depositados sobre la superficie terrestre. (ii) La deposición seca está asociada al transporte en ausencia de precipitación de especies gaseosas y partículas desde la atmósfera hacia la superficie de la localidad (Morselli et al., 2003).

Un porcentaje significativo de los metales pesados es depositado en los alrededores de la fuente que los emite; sin embargo, los aerosoles que por su naturaleza presentan una velocidad de sedimentación pequeña pueden ser transportados por la acción del viento y depositados en lugares distantes del punto de emisión (Smirnioudi et al., 1998). Algunos volátiles como el $\mathrm{Cd}, \mathrm{Zn}, \mathrm{Hg}$ y $\mathrm{Pb}$ pueden ser transportados por gases y cenizas suspendidas en el aire y depositarse en el agua y el suelo. Por lo tanto, estos elementos metálicos pueden ser transportados por el escurrimiento superficial de las cuencas hacia los sistemas de drenaje, arroyos, humedales y ríos, que suelen ser sus principales receptores (Álvarez \& Trento, 2004). También se ha reportado que alrededor del $89 \%$ de los metales pesados se disuelven en el agua de lluvia y, por lo tanto, pueden llegar a la vegetación y a los suelos por procesos que favorecen la ruta de entrada a los organismos vivos (Valenta et al., 1986); una vez en el suelo, los metales pesados pueden quedar retenidos, pero pueden ser movilizados en la solución del suelo mediante mecanismos biológicos y químicos (Pagnanelli et al., 2004).

El objetivo principal de la presente investigación es estudiar el comportamiento de la concentración de los metales pesados asociados con el material atmosférico en suspensión (PM10 y PST) y el sedimento acumulado sobre superficies viales del municipio de Soacha (Colombia). Los metales pesados a evaluar son los siguientes: $\mathrm{Pb}, \mathrm{Cd}$ y Fe. Se pretende estudiar la afinidad de los metales pesados con las fases en suspensión y la sedimentable de superficies viales, y su relación con las condiciones meteorológicas de la zona de investigación.

\section{Materiales y métodos}

\section{Descripción del lugar de investigación}

El área de investigación se localizó en el municipio de Soacha (Colombia). Su clima tropical de montaña (i. e., clima frío) se caracterizó por presentar bajas temperaturas (promedio: $14^{\circ} \mathrm{C}$; variación: $0-18{ }^{\circ} \mathrm{C}$ ), baja humedad y una precipitación media anual de $900 \mathrm{~mm}$. El área de investigación se dividió en dos zonas. La zona 1 correspondió a la localizada en el centro urbano de Soacha, en la vía adyacente al Hospital Central Municipal Mario Gaitán Yanguas (calle 13-carrera 10). La vía cuenta con dos carriles para el tráfico, da acceso a áreas residenciales; sin embargo, es usada ocasionalmente por vehículos de tipo comercial; posee 


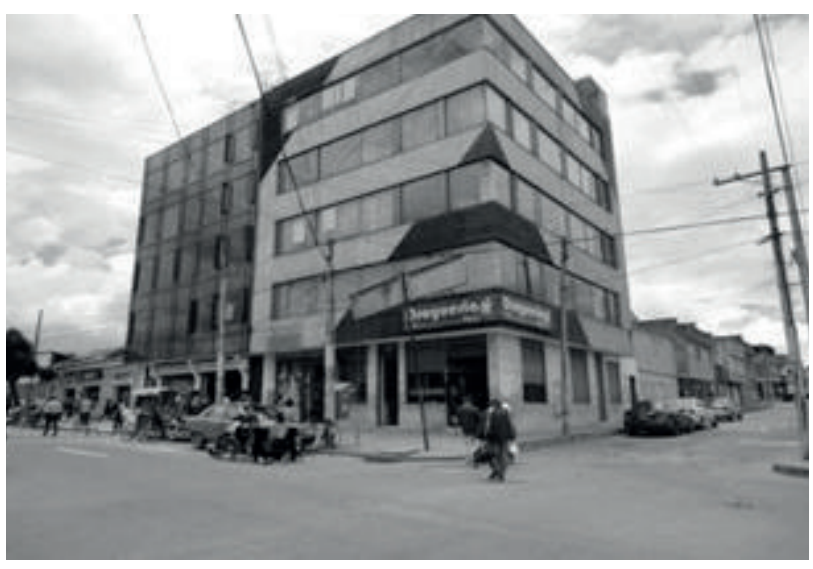

Figura 1. Zona 1 de investigación

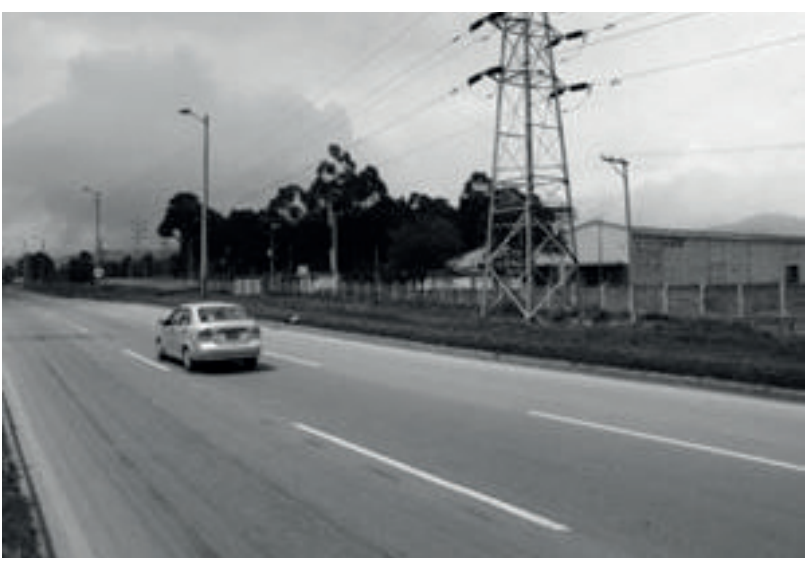

Figura 2. Zona 2 de investigación.

Fuente: elaboración propia.

Fuente: elaboración propia.

\begin{tabular}{|c|c|c|c|}
\hline \multirow[t]{2}{*}{ Características } & \multicolumn{2}{|c|}{ Zona 1: Hospital } & \multirow{2}{*}{$\begin{array}{l}\text { Zona 2: Almacafé } \\
\text { (Autopista Sur) }\end{array}$} \\
\hline & Calle 13 & Carrera 10 & \\
\hline Uso del suelo & Residencial & Residencial & Industrial \\
\hline $\begin{array}{l}\text { Densidad residencial } \\
\text { (habitantes/ha) }\end{array}$ & 600 (alta) & 600 (alta) & 150 (baja) \\
\hline Líneas de tráfico & 2 & 1 & 6 \\
\hline Líneas de parqueo & $1 *$ & Ninguna & Ninguna \\
\hline Pendiente longitudinal (\%) & 0,87 & 0,78 & 0,38 \\
\hline Pendiente transversal (\%) & 4 & 4 & 4 \\
\hline Tipo/estado del pavimento & Asfalto/bueno & Asfalto/deteriorado & Asfalto/bueno \\
\hline $\begin{array}{l}\text { Tráfico promedio diario } \\
\text { (vehículos/día) }\end{array}$ & 1.547 & 1.163 & 40.410 \\
\hline Velocidad promedio $(\mathrm{km} / \mathrm{h})$ & $10-30$ & $10-30$ & $70-90$ \\
\hline Composición del tráfico (\%) & $\begin{array}{l}\text { Automóviles: } 26,2 \text {; vehí- } \\
\text { culos pesados: } 27 \text {; motos: } \\
\text { 13,5; otros: } 33,3\end{array}$ & $\begin{array}{l}\text { Automóviles: } 63,3 \text {; vehí- } \\
\text { culos pesados: } 7,1 \text {; motos: } \\
\text { 10,4; otros: } 19,2\end{array}$ & $\begin{array}{l}\text { Automóviles: } 45 ; \text { vehí- } \\
\text { culos pesados: } 27,4 ; \\
\text { motos y otros: } 27,7\end{array}$ \\
\hline
\end{tabular}

Fuente: elaboración propia.

una elevación media de 2.556 m.s.n.m. (ver figura 1). La zona 2 de investigación se localizó sobre el corredor vial Autopista Sur de Bogotá D.C.Soacha, a la altura de la empresa Almacafé. La vía cuenta en cada uno de sus sentidos con tres carriles para el tráfico y posee una elevación media de 2.564 m.s.n.m. (ver figura 2). La tabla 1 presenta las principales características de cada zona de estudio.

\section{Sistema de muestreo}

Sobre las superficies viales de las dos zonas de investigación se recolectaron muestras de material atmosférico en suspensión y del sedimento acumulado sobre las calzadas. Las muestras del material atmosférico en suspensión y del sedimento viario se recolectaron durante 2010/01/05-2010/05/24. 
De esta manera se recolectaron durante el periodo de investigación 44 muestras de PM10 y 44 muestras de PST; y 88 muestras de sedimento vial para las dos zonas de investigación.

Para la recolección del material atmosférico en suspensión se utilizaron dos estaciones manuales operadas por la Corporación Autónoma Regional de Cundinamarca (CAR): (i) estación fija HospitalSoacha, (ii) estación fija Almacafé-Soacha. En las estaciones se instalaron equipos de alto volumen para la recolección de partículas en suspensión menores de $10 \mu \mathrm{m}$ de diámetro aerodinámico $\left(\mathrm{PM}_{10}\right)$ (ver figura 3$)$ y partículas totales en suspensión (PST). El procedimiento de muestreo se ajustó a lo establecido por la Agencia de Protección Ambiental de EE.UU (EPA/625/R-96/010a-IO-3.1) (EPA, 1999). El periodo de funcionamiento de los equipos manuales de alto volumen fue de 24 horas. Adicionalmente, al inicio y al final del periodo diario de muestreo se registró la hora y fecha, la temperatura y la presión atmosférica. Finalmente, los filtros fueron retirados de los equipos al final de cada periodo diario de muestreo, se doblaron a la mitad y se depositaron dentro de un sobre ajustado, limpio y debidamente identificado para su posterior análisis químico (i. e., determinación de metales pesados).

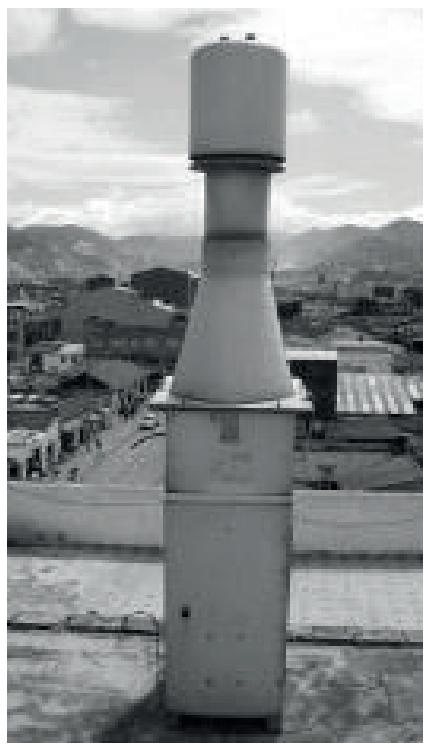

Figura 3. Sistema manual de muestreo de $\mathrm{PM}_{10}$.

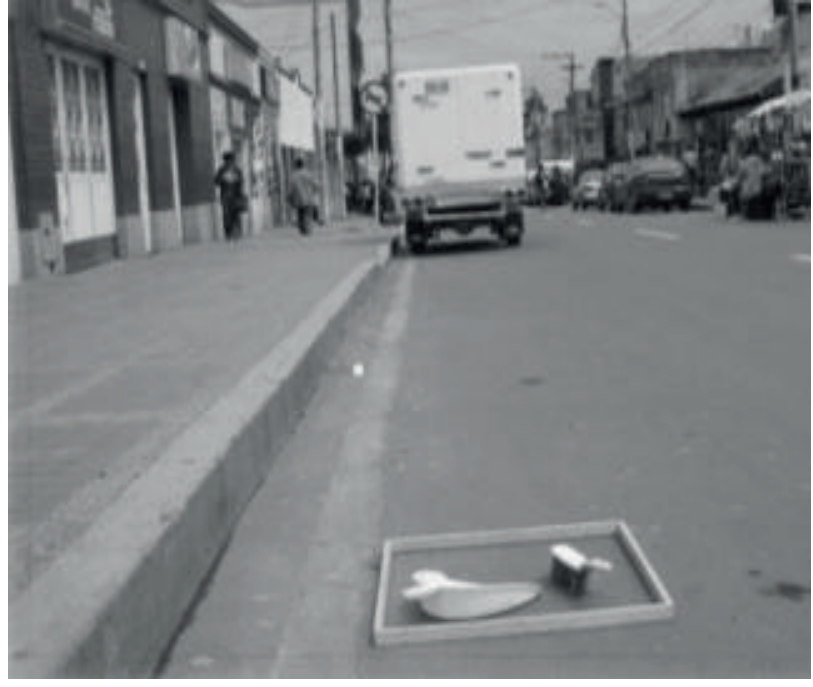

Figura 4. Sistema de muestreo del sedimento vial.

Fuente: elaboración propia.

La superficie de muestreo para la recolección del sedimento vial se controló mediante un marco de madera de $0,50 \times 0,50 \mathrm{~m}\left(0,25 \mathrm{~m}^{2}\right)$. El sedimento se recolectó a través de un barrido directo de la superficie de la calzada con un cepillo de cerdas finas de plástico (i. e., desde los bordes hacia el centro del marco) y con un recogedor manual de plástico (ver figura 4). Los lugares de recolección del sedimento fueron marcados con pintura con el objeto de impedir repeticiones sobre la superficie en posteriores muestreos. Finalmente, el sedimento vial fue almacenado en bolsas plásticas herméticas y debidamente identificadas para su posterior análisis químico (determinación de metales pesados).

\section{Análisis de laboratorio}

Inicialmente se realizó un retratamiento a las muestras del material atmosférico en suspensión y del sedimento vial; consistió en un secado a partir del procedimiento establecido para la determinación de humedad con base en la norma ASTM D2216 (ASTM, 2000). Posteriormente, a las muestras del sedimento vial se les realizó un tamizado para separar la fracción de tamaño menor de 250 $\mu \mathrm{m}$, para someterla al análisis químico de determinación del contenido de elementos metálicos. 
El método de extracción para filtros de $\mathrm{PM}_{10}$ y PST se basó en lo establecido por la Agencia de Protección Ambiental de EE.UU (EPA/625/R96/010a-IO-3.1) (EPA, 1999). Por otro lado, la extracción de metales pesados para el sedimento vial se realizó a partir de lo establecido en la norma ISO 11466 (ISO, 2000). A partir del extracto obtenido, se determinó el contenido de Fe utilizando un equipo (Única modelo 989) de espectrometría de absorción atómica de llama (ISO 11047) (ISO, 2000), y se utilizó un equipo de plasma inductivamente acoplado con espectrometría de masas (ICP-MS Varían 820) para la determinación de $\mathrm{Pb}$ y Cd (ISO 11047) (ISO, 2000).

Finalmente, los datos climatológicos de precipitación, temperatura, y dirección y velocidad del viento fueron obtenidos de cuatro estaciones operadas por la Corporación Autónoma Regional de Cundinamarca (CAR): El Fute, Paraíso Perdido, Almacafé y Hospital; y de tres estaciones operadas por la Secretaría Distrital de Ambiente de la ciudad de Bogotá (SDA): Carvajal, Kennedy y Tunal.

\section{Resultados y discusión}

\section{Descripción climática del área de investigación}

Inicialmente se estudió la variación temporal de la precipitación y la temperatura durante el periodo de investigación (2010/01/05-2010/05/24). Con el objeto de tener una visión temporal más completa se procedió a ampliar el periodo de análisis para los parámetros climatológicos: 2009/06/01-2010/06/01 (ver figura 5). Como se pudo observar, la variación temporal de la precipitación presentó dos periodos de aumento: (i) entre los meses de octubre y noviembre, (ii) entre abril y mayo; en este sentido, la precipitación total acumulada durante el segundo periodo fue 1,26 veces superior. Por otro lado, existieron dos periodos de disminución de la precipitación: (i) entre los meses de junio y septiembre y (ii) entre diciembre y marzo; en este sentido, la precipitación total acumulada en el segundo periodo fue 2,30 veces inferior. Como era de esperar, en este segundo periodo de tiempo seco existió el mayor incremento en la temperatura del área de investigación: promedio en marzo de $17,4^{\circ} \mathrm{C}$ (ver figura 5). La temperatura promedio durante el periodo de investigación fue de $15,0^{\circ} \mathrm{C}$. Algunos investigadores han reportado una tendencia similar entre la precipitación y la temperatura (p.ej., Esquivel, 2007).

Por otro lado, la velocidad y la dirección del viento en el periodo de investigación presentaron valores dominantes entre 2,1 y $3,6 \mathrm{~m} / \mathrm{s}$ y desde el sur, respectivamente; en este sentido, en los meses de agosto y septiembre la velocidad del viento aumentó, y en abril y mayo disminuyó; este último periodo coincidió con los registros más elevados de precipitación (ver figura 5).

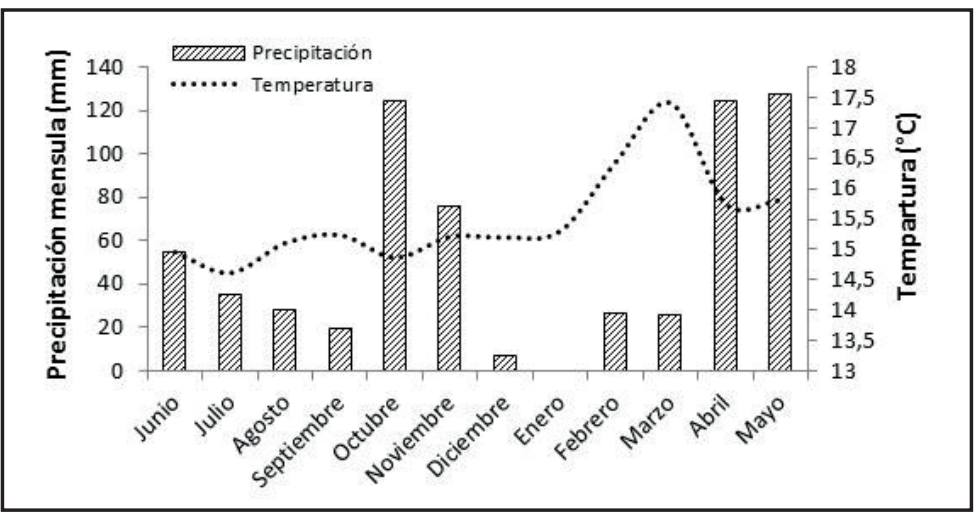

Figura 5. Precipitación total y temperatura promedio mensual en el área de investigación.

Fuente: elaboración propia. 
Finalmente, durante el periodo de investigación (2010/01/05-2010/05/24) se registró una precipitación acumulada de $66,5 \mathrm{~mm}$ en la estación Hospital (zona 1) y de $72,5 \mathrm{~mm}$ en la estación Almacafé (zona 2); en promedio para las dos estaciones correspondió a un $47 \%$ de la precipitación total anual (i.e., entre 2009/06/01-2010/06/01). La temperatura promedio fue de $16,5^{\circ} \mathrm{C}$, con mínimas de $13,4{ }^{\circ} \mathrm{C}$ (mayo) y máximas de $18,5^{\circ} \mathrm{C}$ (marzo). La velocidad media del viento fue de $2,4 \mathrm{~m} / \mathrm{s}$ con velocidades mínimas de $1 \mathrm{~m} / \mathrm{s}$ (enero y febrero) y máximas de $4,4 \mathrm{~m} / \mathrm{s}$ (abril). Por último, durante el mes de enero no se registraron precipitaciones, lo cual coincidió con un aumento de la temperatura hasta el mes de marzo.

\section{Concentración de metales pesados}

Las concentraciones más elevadas de metales pesados en el sedimento vial se registraron para Fe y las más bajas para Cd (ver tabla 2). Las concentraciones de $\mathrm{Pb}$ y $\mathrm{Cd}$ tendieron a aumentar durante los meses de enero y febrero; adicionalmente, las concentraciones de estos metales pesados tendieron a disminuir durante los meses de abril y mayo. A partir de lo anterior, los resultados sugirieron que las concentraciones de los elementos metálicos en estudio tendieron a aumentar en tiempo seco y a disminuir en época de lluvia (ver figura 6).

Con respecto al material atmosférico en suspensión (i. e., $\mathrm{PM}_{10}$ y PST), se observó que Fe fue el elemento metálico que registró las mayores concentraciones (ver tabla 2) y Cd fue el metal pesado que registró las menores concentraciones. Lo anterior, coincidiendo con la tendencia observada en el sedimento vial. Por lo tanto, los resultados indicaron una tendencia similar en la distribución de la concentración metálica en áreas aledañas a superficies viales; es decir, en el material atmosférico en suspensión y el sedimento depositado sobre la vía.

\begin{tabular}{|c|c|c|c|c|}
\hline \multirow{2}{*}{$\begin{array}{l}\bar{\pi} \\
\stackrel{\pi}{0} \\
\end{array}$} & \multirow{2}{*}{$\begin{array}{l}0 \\
\stackrel{0}{0} \\
\end{array}$} & \multirow{2}{*}{$\begin{array}{l}\text { Sedimento } \\
\text { vial } \\
<250 \mu \mathrm{m}\end{array}$} & \multicolumn{2}{|c|}{ Material atmosférico } \\
\hline & & & $\mathrm{PM}_{10}$ & PST \\
\hline $\mathrm{Pb}$ & $\begin{array}{l}1 \\
2\end{array}$ & $\begin{array}{l}79 \pm 21 \\
199 \pm 28\end{array}$ & $\begin{array}{l}1818 \pm 473 \\
2835 \pm 613\end{array}$ & $\begin{array}{l}864 \pm 184 \\
866 \pm 604\end{array}$ \\
\hline $\mathrm{Cd}$ & $\begin{array}{l}1 \\
2\end{array}$ & $\begin{array}{l}0,65 \pm 0,4 \\
0,48 \pm 0,2\end{array}$ & $\begin{array}{l}5,74 \pm 2,1 \\
1,52 \pm 1,2\end{array}$ & $\begin{array}{l}1,36 \pm 0,6 \\
1,01 \pm 0,8\end{array}$ \\
\hline $\mathrm{Fe}^{\mathrm{a}}$ & $\begin{array}{l}1 \\
2\end{array}$ & $\begin{array}{l}8,79 \pm 0,7 \\
20,11 \pm 1,8\end{array}$ & $\begin{array}{l}10,52 \pm 2,7 \\
9,42 \pm 2,5\end{array}$ & $\begin{array}{l}9,33 \pm 1,8 \\
9,09 \pm 1,5\end{array}$ \\
\hline
\end{tabular}

Fuente: elaboración propia.

Al comparar las concentraciones metálicas entre $\mathrm{PM}_{10}$ y PST, se evidenciaron magnitudes más elevadas en la fracción potencialmente respirable $\left(\mathrm{PM}_{10}\right)$, lo que sugiere a este parámetro de calidad del aire como un indicador de la presencia de

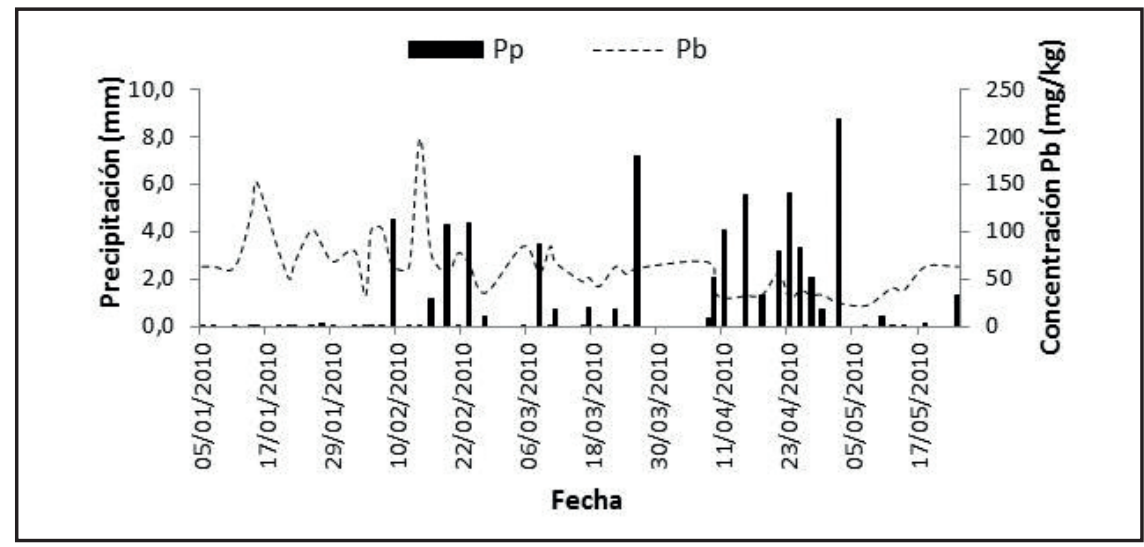

Figura 6. Concentración de Pb versus la precipitación durante el periodo de investigación en la estación Hospital (zona 1).

Fuente: elaboración propia. 
metales pesados en suspensión en áreas aledañas a las superficies viales (ver tabla 2). Lo anterior, probablemente debido a que las fuentes de los elementos metálicos estuvieron asociadas con tamaños de partícula menores o iguales de $10 \mu \mathrm{m}$. En este sentido, las concentraciones metálicas asociadas con $\mathrm{PM}_{10}$ fueron en promedio 2,21 veces superiores a las registradas por PST (i. e., para $\mathrm{Pb}, \mathrm{Cd} y$ $\mathrm{Fe})$. Adicionalmente, las concentraciones de $\mathrm{Pb}$ y
Cd registradas por la fracción potencialmente respirable $\left(\mathrm{PM}_{10}\right)$ fueron en promedio 17,6 y 4,7 veces superiores a las registradas por el sedimento vial. Sin embargo, Fe presentó una tendencia opuesta en la zona 2 de investigación; es decir, se registraron mayores concentraciones metálicas en el sedimento vial; lo anterior, probablemente estuvo asociado al aporte de las fuentes móviles (tráfico promedio: 40.410 vehículos/día) y al tamaño de

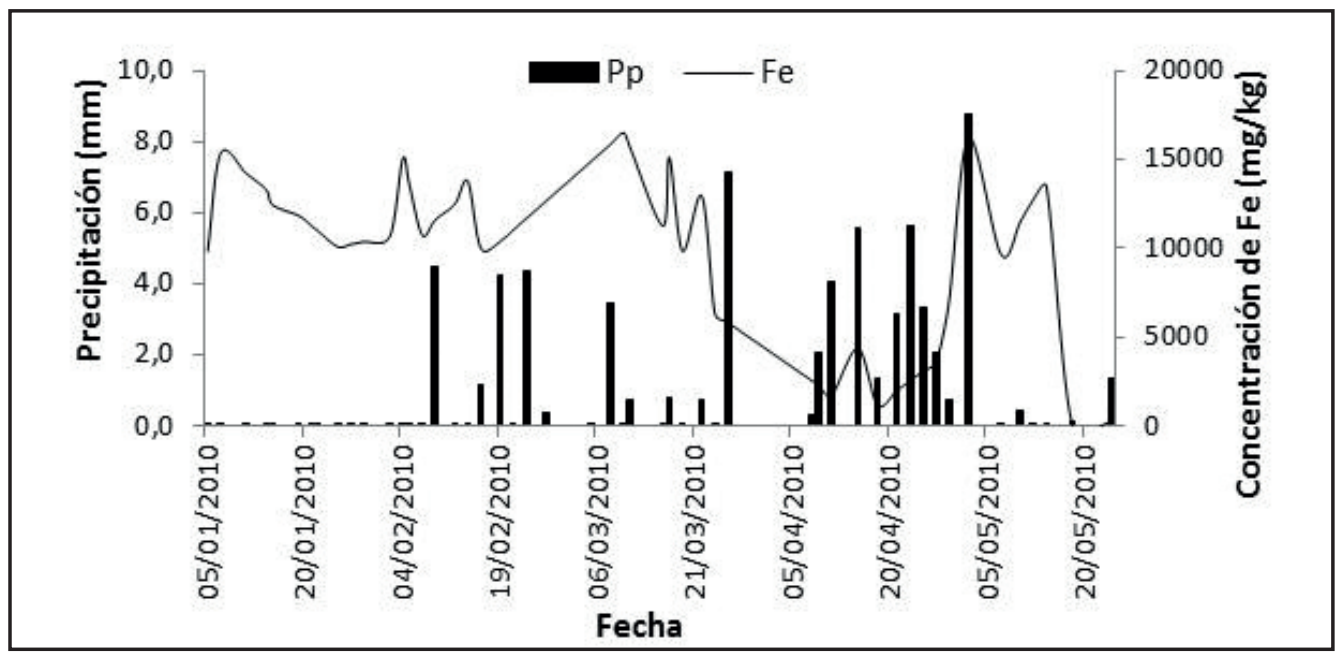

Figura 7. Concentración de Fe versus la precipitación durante el periodo de investigación en la estación Almacafé (zona 2).

Fuente: elaboración propia.

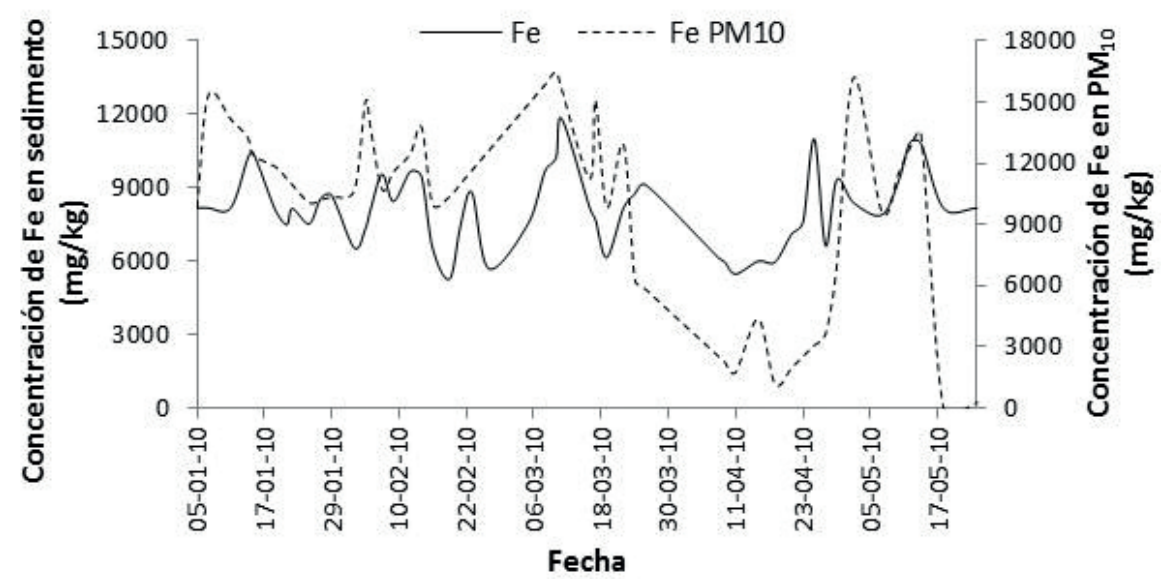

Figura 8.Variación temporal de la concentración de Fe asociada con PM10 y el sedimento vial de la estación Almacafé (zona 2). 
partícula asociado con las fuentes de contaminación (i. e., entre 10 y $250 \mu \mathrm{m}$ ).

Al evaluar la relación entre las variables climáticas y la concentración de metales pesados asociados con el material atmosférico en suspensión, se observó que las concentraciones de Fe tendieron a disminuir durante el mes de abril, en coincidencia con la época de aumento de las precipitaciones (ver figura 7). En este sentido, los coeficientes de correlación lineal de Pearson mostraron para $\mathrm{Fe}, \mathrm{Pb}$ y Cd una correlación negativa media $(\mathrm{r}=0,51)$, débil $(r=0,39)$ y débil $(r=0,30)$ entre la magnitud de la precipitación y la concentración metálica, respectivamente. Lo anterior coincide con la tendencia observada en el sedimento vial. Nuevamente, los resultados indicaron una tendencia similar en la distribución de la concentración metálica en áreas aledañas a superficies viales; es decir, en el material atmosférico en suspensión y el sedimento depositado sobre las vías.

A partir de la similitud detectada en la variación temporal de la concentración de Fe asociada con $\mathrm{PM}_{10}$ y el sedimento vial, se procedió a estudiar su relación a través del coeficiente de correlación lineal de Pearson (r). Los resultados mostraron en promedio la existencia de una correlación positiva media entre las concentraciones de $\mathrm{Fe}(\mathrm{r}=0,51)$ y $\mathrm{Pb}(\mathrm{r}=0,66)$ asociadas con $\mathrm{PM}_{10}$ y el sedimento vial de las áreas de investigación (ver figura 8). Por otro lado, las concentraciones de Cd presentaron una correlación positiva débil $(\mathrm{r}=0,26)$ entre $\mathrm{PM}_{10}$ y el sedimento vial.

Al comparar las concentraciones metálicas registradas en las dos áreas de investigación, se observó que la zona 2 (Almacafé-Autopista Sur) tendió a presentar las mayores concentraciones de $\mathrm{Pb}$ y $\mathrm{Fe}$ asociadas con el sedimento vial (ver tabla 2), excepto para $\mathrm{Cd}$, en que las mayores concentraciones se presentaron en la zona 1 (Hospital-área urbana). Por lo tanto, los resultados sugirieron que el uso del suelo (industrial-comercial) y la elevada densidad de tráfico (40.410 vehículos/día) de la zona 2 fueron los principales condicionantes para el aumento de las concentraciones de $\mathrm{Pb}$ y $\mathrm{Fe}$ asociadas con el sedimento vial (ver tabla 1). Con respecto al material atmosférico en suspensión, la zona 2 tendió a registrar las mayores concentraciones de $\mathrm{Pb}$; sin embargo, registró concentraciones de $\mathrm{Fe}$ y $\mathrm{Cd}$ ligeramente inferiores a las detectadas en la zona 1: 1,12 y 1,03 veces, respectivamente (ver tabla 2).

A partir de la anterior tendencia, se procedió a utilizar la prueba t de Student con el objeto de evaluar la afinidad en el origen de los elementos metálicos en las dos áreas de investigación (zona 1: Hospitalárea urbana; zona 2: Almacafé-Autopista Sur). Los resultados mostraron que no existieron diferencias significativas en las concentraciones de $\mathrm{Cd}$ entre las zonas 1 y 2 . Por consiguiente, se sugirió que las concentraciones de $\mathrm{Cd}$ provinieron de las mismas fuentes; por ejemplo, fundiciones y acerías, talleres y garajes, chatarrerías, e industrias del plástico, pintura, lacados y curtidos; todas presentes en la municipalidad de investigación (AMS, 2008). Por otro lado, para el material en suspensión $\left(\mathrm{PM}_{10} \mathrm{y}\right.$ PST) la prueba t de Student mostró que existieron diferencias significativas en la concentración entre las zonas 1 y 2 , lo cual evidencia que los elementos metálicos en estudio no provinieron de la misma fuente contaminante (fijas o móviles).

\section{Comparación con la normatividad}

Actualmente, no existe legislación colombiana que limite las concentraciones másicas de los metales pesados en suelos, para la protección de la salud humana por inhalación de partículas de suelo, inhalación de vapores del suelo en el exterior, ingestión de suelo y contacto dérmico con el suelo. Entonces, se procedió a comparar las concentraciones registradas con respecto a la legislación del País Vasco, España (PV, 2005) (ver tabla 3). Como se pudo observar, con respecto al sedimento vial, las concentraciones de $\mathrm{Pb}$ en la zona 2 superaron en 1,33 veces los límites establecidos por ingestión y contacto dérmico con el suelo. Con respecto a la inhalación de partículas y de vapores del suelo en el exterior, se observó que el $\mathrm{Pb}$ en las zonas 1 y 2 superó los límites establecidos en 12,1 y 18,9 veces, respectivamente. Finalmente, es importante señalar que las concentraciones metálicas en suspensión se obtuvieron en áreas aledañas a las superficies viales. 


\begin{tabular}{|c|c|c|c|c|}
\hline \multicolumn{2}{|l|}{ Lugar } & $\begin{array}{c}\mathrm{Pb} \\
(\mathrm{mg} / \mathrm{kg})\end{array}$ & $\begin{array}{c}\mathrm{Cd} \\
(\mathrm{mg} / \mathrm{kg})\end{array}$ & $\begin{array}{c}\mathrm{Fe} \\
(\mathrm{mg} / \mathrm{kg})\end{array}$ \\
\hline \multicolumn{2}{|c|}{$\begin{array}{l}\text { Límite (País Vasco, } \\
\text { España) }\end{array}$} & 150 & 8 & - \\
\hline \multirow[t]{2}{*}{$\begin{array}{l}\text { Sedimento } \\
\text { vial }\end{array}$} & $\begin{array}{c}\text { Zona } \\
1\end{array}$ & 79 & 0,65 & 8.790 \\
\hline & $\begin{array}{c}\text { Zona } \\
2\end{array}$ & 199 & 0,48 & 20.110 \\
\hline \multirow[t]{2}{*}{$\mathrm{PM}_{10}$} & $\begin{array}{c}\text { Zona } \\
3\end{array}$ & 1.818 & 5,74 & 10.520 \\
\hline & $\begin{array}{c}\text { Zona } \\
4\end{array}$ & 2.835 & 1,52 & 9.420 \\
\hline \multicolumn{5}{|c|}{$\begin{array}{l}\text { a Límites por inhalación, ingestión y contacto dér- } \\
\text { mico con el suelo. }\end{array}$} \\
\hline
\end{tabular}

Fuente: elaboración propia.

\section{Conclusiones}

Los resultados muestran para el material atmosférico en suspensión y el sedimento vial el siguiente orden en la magnitud de la concentración metálica asociada $(\mathrm{mg} / \mathrm{kg}): \mathrm{Fe}>\mathrm{Pb}>\mathrm{Cd}$. Lo anterior sugiere una tendencia similar en la distribución de los metales pesados presentes en las áreas aledañas a las superficies viales (i. e., para el material en suspensión y el sedimentado). Así mismo, las concentraciones de $\mathrm{Pb}$ y $\mathrm{Cd}$ asociadas con el material atmosférico en suspensión y el sedimento vial tienden a aumentar en tiempo seco y a disminuir en época de lluvias.

Por otro lado, las concentraciones metálicas asociadas con la fracción potencialmente respirable $\left(\mathrm{PM}_{10}\right)$ son en promedio 2,21 veces superiores a las registradas por PST (para $\mathrm{Pb}, \mathrm{Cd}$ y $\mathrm{Fe}$ ). Adicionalmente, las concentraciones de $\mathrm{Pb}$ y $\mathrm{Cd}$ registradas para $\mathrm{PM}_{10}$ son en promedio 17,6 y 4,7 veces superiores a las registradas por el sedimento vial.

Al comparar las concentraciones metálicas registradas en las dos áreas de investigación, los resultados sugieren que el uso del suelo (industrial-comercial) y la elevada densidad de tráfico (40.410 vehículos/ día) de la zona 2 son los principales condicionantes para el aumento de las concentraciones de $\mathrm{Pb}$ y Fe asociadas con el sedimento vial.

Con respecto a la legislación del País Vasco, las concentraciones de $\mathrm{Pb}$ en la zona 2 superan en 1,33 veces los límites establecidos para ingestión y contacto dérmico con el suelo. Con respecto a la inhalación de partículas y de vapores del suelo en el exterior, se observa que el $\mathrm{Pb}$ en las zonas 1 y 2 supera los límites establecidos en 12,1 y 18,9 veces, respectivamente.

Finalmente, los resultados permiten profundizar el conocimiento acerca de la presencia y el comportamiento de los metales pesados asociados con las partículas del material atmosférico en suspensión y el material acumulado sobre las superficies viales de la localidad de Soacha. Lo anterior, con el objeto de que las instituciones públicas encargadas de la gestión de la contaminación ambiental en la municipalidad puedan diseñar, implementar y mejorar los sistemas de control de la contaminación por metales pesados.

Agradecimientos: los autores agradecen el apoyo financiero brindado por el Centro de Investigación y Desarrollo Científico de la Universidad Distrital Francisco José de Caldas (contrato 31 de 2010), la Corporación Autónoma Regional de Cundinamarca (CAR) y el Grupo de Investigación en Ingeniería Ambiental de la Universidad Distrital Francisco José de Caldas (GIIAUD).

\section{Referencias}

Álvarez, A. \& Trento, A. (2004). Transporte de metales pesados en cursos fluviales. Mecánica Computacional, 23(1), 1151-1165.

Amato, F., Pandolfi, M., Viana, M., Querol, X., Alastuey, A., \& Moreno, T. (2009). Spatial and chemical patterns of PM10 in road dust deposited in urban environment. Atmospheric Environment, 43(9), 1650-1659.

AMS. (2008). Diagnóstico ambiental y agropecuario. Soacha: Dirección Gestión Bioambiental 
y Asistencia Técnica Agropecuaria, Alcaldía Municipal de Soacha.

AMS. (2008). Plan de Desarrollo 2008-2011. Soacha para vivir mejor. Soacha: Alcaldía Municipal de Soacha.

AMS. (2009). Perfil ambiental municipal. Visión comunitaria. Soacha: Alcaldía Municipal de Soacha.

ASTM. (2000). Standard test method for laboratory determination of water (moisture) content of soil and rock by mass (ASTM D2216). West Conshohocken: American Society for Testing and Materials.

Bustos, C. (2004). Aplicación de modelos de dispersión atmosférica en la evaluación de impacto ambiental: análisis del proceso. Santiago de Chile: Universidad de Chile.

PV. (2005). Ley 1/2005: Prevención de la contaminación del suelo del País Vasco. Bilbao: Boletín Oficial del País Vasco, Eusko Legebiltzarra.

EPA. (1999). Sampling of ambient air for total suspended particulate matter SPM and PM10 using High Volume HV sampler. Washinton D.C.: Center for Environmental Research Information, Office of Research and Development, U.S. Environmental Protection Agency.

Esquivel, E. (2007). Lluvia y sequía en el norte de México. Un análisis de la precipitación histórica en Chihuahua. Gaceta Ecológica, 65, 2442.

Ferré-Huguet, N., Schuhmacher, M., Llobet, J.\& Domingo, J. (2007). Metales pesados y salud. Mapfre Seguridad, 108(4), 50-58.

García, R. (2007). Determinación de metales pesados en la precipitación pluvial de una zona urbana (Ciudad de México) y de una zona rural (Rancho Viejo, Edo. de México).México D.F.: Universidad Nacional Autónoma de México.
ISO. (2000). Soil quality, determination of cadmium, chromium, cobalt, copper, lead, manganese, nickel and zinc, flame and electrothermal atomic absorption spectrometric methods (ISO 11047). Geneva: International Organization for Standardization.

ISO. (2000). Soil quality, extraction of trace elements soluble in aqua regia (ISO 11466). Geneva: International Organization for Standardization.

Juda-Rezler, K., Reizer, M., \& Oudinet, J. P. (2011). Determination and analysis of PM10 source apportionment during episodes of air pollution in central eastern european urban areas: The case of wintertime 2006. Atmospheric Environment, 45(36), 6557-6566.

Morselli, L., Olivieri, P., Brusori, B., \& Passarini, F. (2003). Soluble and insoluble fractions of heavy metals in wet and dry atmospheric depositions in Bologna, Italy. Environmental Pollution, 124(3), 457-469.

Olivares, G. (2001). Dispersión regional de azufre oxidado en Chile central. Santiago de Chile: Universidad de Chile.

OMS. (2004). Guías para la calidad del aire. Lima: Departamento de Protección del Medio Humano, Programa de Salud Ocupacional, Organización Mundial de la Salud.

Pachón, J., Bustos, M., García, H., Bravo, H.\& Sosa, R. (2005). Determinación de metales pesados en partículas PM10 en la atmósfera de la zona metropolitana de la ciudad de Bogotá (Colombia).Boletín Redaire, 16, 52-65.

Pagnanelli, F., Moscardini, E., Giuliano, V., \& Toro, L. (2004). Sequential extraction of heavy metals in river sediments of an abandoned pyrite mining area: Pollution detection and affinity series. Environmental Pollution, 132(2), 189201. 
Pérez, G. (2005). Disponibilidad de metales tóxicos en sitios contaminados. Aplicaciones y limitaciones de la fraccionación en la determinación de gradientes de polución. Barcelona: Universidad Autónoma de Barcelona.

Smirnioudi, V., Thomaidis, M., Piperaki, E., \& Siskos, P. (1998). Determination of trace metals in wet and dust deposition in Greece. Fresenius Environmental Bulletin, 7(1-2), 85-90.

Turtós, I., Meneses, E.\& Díaz, N. (2003). Dispersión local de contaminantes atmosféricos producto de la generación eléctrica. Caso de estudio: Central termoeléctrica Carlos Manuel de Céspedes de Cienfuegos. La Habana: Centro de Gestión de la Información y Desarrollo de la Energía.
Valenta, P., Nguyen, V., \& Nornberg, H. (1986). Acid and heavy metal pollution by wet deposition. Science of the Total Environment, 55(11), 311-320.

Zafra, C. A., Temprano, J., \& Tejero, I. (2011). Distribution of the concentration of heavy metals associated with the sediment particles accumulated on road surfaces. Environmental Technology, 32(9), 997-1008.

Agradecimientos: proyecto de investigación financiado por el Centro de Investigación y Desarrollo Científico de la Universidad Distrital Francisco José de Caldas. Grupo de Investigación en Ingeniería Ambiental (GIIAUD) 